Association for Information Systems AIS Electronic Library (AISeL)

Wirtschaftsinformatik Proceedings 1999

Wirtschaftsinformatik

February 1999

\title{
Die Beraterbank im Internet: Verstärkung der Kundenbindung durch individuelle Finanzdienstleistungen
}

Hans-Dieter Groffmann

BROKAT Infosystems AG, Stuttgart, hdgroffmann@brokat.com

Vera Schäfers

IDS Scheer AG, Saarbrücken, v.schaefers@ids-scheer.de

Sven Viktorin

BROKAT Infosystems AG, Stuttgart, sviktorin@brokat.com

Follow this and additional works at: http://aisel.aisnet.org/wi1999

\section{Recommended Citation}

Groffmann, Hans-Dieter; Schäfers, Vera; and Viktorin, Sven, "Die Beraterbank im Internet: Verstärkung der Kundenbindung durch individuelle Finanzdienstleistungen" (1999). Wirtschaftsinformatik Proceedings 1999. 14.

http://aisel.aisnet.org/wi1999/14

This material is brought to you by the Wirtschaftsinformatik at AIS Electronic Library (AISeL). It has been accepted for inclusion in Wirtschaftsinformatik Proceedings 1999 by an authorized administrator of AIS Electronic Library (AISeL). For more information, please contact elibrary@aisnet.org. 


\section{Die Beraterbank im Internet: Verstärkung der Kundenbindung durch individuelle Finanzdienstleistungen}

Hans-Dieter Groffmann

BROKAT Infosystems AG, Stuttgart (hdgroffmann@ brokat.com)

Vera Schäfers

IDS Scheer AG, Saarbrücken (v.schaefers@ids-scheer.de)

Sven Viktorin

BROKAT Infosystems AG, Stuttgart, (sviktorin@brokat.com)

\section{Inhalt}

1 Internet-Beratung: Neue Vertriebswege für individuelle Finanzdienstleistungen

2 Ziel der Internet-Beratung: Verstärkung der Kundenbindung

3 Prototyp einer Internet-Kundenberatung

4 Module einer interaktiven Internet-Beratung

4.1 Daten-Konferenz-Modul

4.1.1 Chat Systeme

4.1.2 Whiteboard

4.1.3 Application Sharing

4.2 Audiomodul

4.3 Videomodul

4.4 Call-Me Button

5 Aufbau einer sicheren und flexiblen Lösung

5.1 E-Services-Plattform als zentrales Element

5.1.1 Sicherheitskonzept

5.1.2 Sicherheits-Architektur

5.2 Architektur eines Internet-Call-Centers

6 Beraterbank-Paket 


\begin{abstract}
Einen wesentlichen Vertriebskanal für Finanzdienstleister stellt heutzutage das Internet dar. Internet Banking ist daher nicht mehr eine Frage des "Ob" oder des "Wann" sondern eine schlichte Frage des Überlebens. Nachdem führende Finanzdienstleister schon seit geraumer Zeit das Internet als Informations- und Transaktionskanal einsetzen, ist es an der Zeit die nächste Stufe im Internet Banking einzuführen: Die Beraterbank. Nur mit einem derartigen Ansatz wird es Finanzdienstleistern zukünftig möglich sein, ihre Kunden langfristig zu binden bzw. sich von Mitbewerbern abzusetzen. Der folgende Beitrag stellt einen Prototypen für die Kundenberatung via Internet vor und beschreibt die Module einer interaktiven Internet-Beratung unter besonderer Berücksichtigung der Sicherheitsaspekte.
\end{abstract}

\title{
1 Internet-Beratung: Neue Vertriebswege für individuelle Finanzdienstleistungen
}

Die technischen Entwicklungen der letzten zwanzig Jahre haben zahlreiche neue Wege zur Kommunikation zwischen Kunde und Bank ermöglicht. Das direkte, persönliche Gespräch in der Bank wird schrittweise durch den Kontakt über Medien wie Telefon, Fax, SB Automaten, Multimedia-Kioske, Onlinedienste und Videoconferencing ergänzt bzw. abgelöst (Birkelbach 1998; Disterer/ReimersMortensen 1997). Neben diesen technischen Herausforderungen müssen sich Finanzdienstleister gestiegenen Kundenanforderungen an Flexibilität, Service und Performance stellen (Scholz et al. 1995). "Banking anytime, anywhere, by any mean" ist die Maxime.

Aufgrund dieser Entwicklungen sind entscheidende Veränderungen in der Bedeutung der verschiedenen Vertriebskanäle für Finanzdienstleister zu erwarten. Innerhalb der nächsten zehn Jahre wird sich das Internet zum wichtigsten bankbetrieblichen Absatzkanal entwickeln (Lange 1998, S. 21). Untersuchungen von Booz Allen \& Hamilton lassen erwarten, daß die Bedeutung einer Filiale bisheriger Prägung in Europa hinter das Telefon-Banking auf den dritten Platz zurückfällt, während sie in den USA nur noch an achter Stelle stehen wird (Booz Allen \& Hamilton 1997).

Inzwischen ist fast jede Bank und bereits viele Versicherungsunternehmen im Internet präsent. Das derzeitige Dienstleistungsspektrum der Finanzdienstleister im Internet ist kaum auf Beratung ausgerichtet, sondern erstreckt sich bislang hauptsächlich auf Informations- und Finanztransaktionsangebote. Hierbei setzen sich die Informationsangebote aus allgemeinen Informationsseiten und interaktiven Informationsangeboten zusammen. Allgemeine Informationsseiten sollen dem Internet-User einen Überblick über das Kreditinstitut und sein Dienstleistungsspektrum geben. Damit Kunden nicht nur einmal, sondern 
regelmäßig die Seiten der Bank aufrufen, werden diese häufig um Zusatzinformationen, wie z. B. Börsen-News ergänzt.

Bei interaktiven Informationsangeboten liegt der Individualisierungsgrad der zur Verfügung gestellten Informationen wesentlich höher. Vorausgesetzt wird jedoch, daß der Kunde Angaben über sich selbst und seine Präferenzen macht. Ein typisches Beispiel hierfür ist die Berechnung eines Bausparvertrages, bei dem der Kunde den Sparbeitrag sowie die gewünschte Laufzeit festlegen kann.

Die angebotenen Finanztransaktionen lassen sich in die Abwicklung des Zahlungsverkehrs (z. B. Überweisung, Dauerauftrag) sowie in die Abwicklung sonstiger Finanzdienstleistungen (z. B. Wertpapier-Order, Depotübersicht) unterteilen. Diese Transaktionen werden inzwischen von den meisten Banken angeboten und stellen somit keinen besonders geeigneten Differenzierungsfaktor mehr dar.

\section{Dienstleistungsangebot der Banken im Internet}

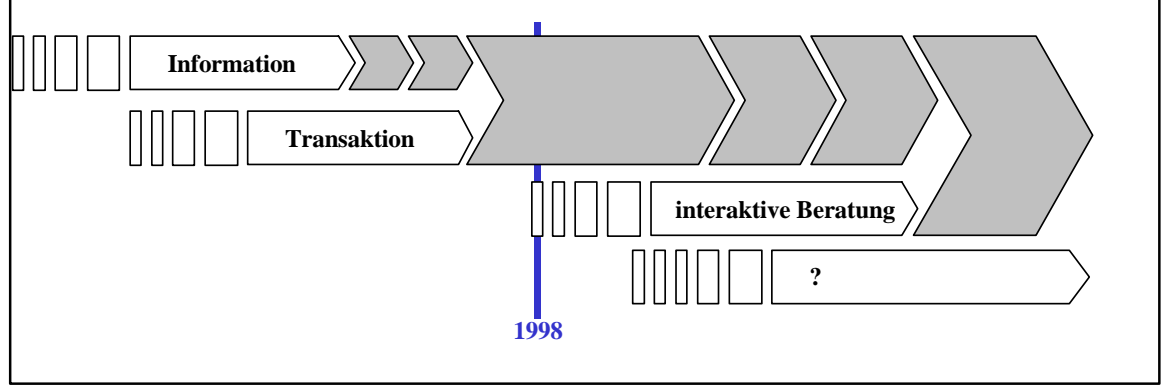

Abbildung 1: Dienstleistungsangebot der Banken im Internet

Einen echten Differenzierungsfaktor stellt hingegen die interaktive Beratung dar, welche heutzutage bei den Banken noch kaum eingesetzt wird. Die Gründe hierfür wurden in der Vergangenheit an zwei Faktoren festgemacht:

- Fehlen des direkten Kontaktes zwischen Kunde und Finanzdienstleister,

- Fragen der Sicherheit (Unisys 1996).

Beide Faktoren können heute nicht mehr als Hinderungsgrund angeführt werden, da neue Technologien sowohl eine individuelle Beratung über das Medium Internet (Dohmen 1998; Knierim 1997; Radetzky 1998) als auch eine ausreichende Gewährleistung der Sicherheit von Transaktionen ermöglichen. 


\section{Ziel der Internet-Beratung: Verstärkung der Kundenbindung}

Eines der Hauptziele, welches Finanzinstitute mit der interaktiven Beratung verfolgen ist die Stärkung der Kundenbindung. Diese zentrale Zielgröße dient sowohl zur Generierung von Erträgen als auch zur Erhöhung der Profitabilität. Kundenbindung sorgt für Cross Selling, für vergleichsweise niedrige Beratungskosten, für eine geringere Abwanderungsgefährdung und für Weiterempfehlungen im Familien- und Freundeskreis (Lange 1998, S. 88).

Eine wesentliche Rolle beim Aufbau und bei der Erhaltung langfristiger Kundenbeziehungen spielen neben der Kundenzufriedenheit persönliche Kontakte und individuelles Marketing. Vor allem die persönliche Kommunikation trägt bei Finanzdienstleistungen aufgrund ihrer größeren Glaubwürdigkeit und Individualität in der Kommunikation zur Beziehungspflege bei.

Üblich ist in diesem Bereich bislang nur die Anforderung von Informationen per E-Mail und/oder die Möglichkeit der telefonischen Kontaktaufnahme. Weiterhin wird versucht, häufig gestellte Kundenfragen im Rahmen des Informationsangebotes im Internet (z. B. über FAQ-Listen) zu beantworten.

\section{Prototyp einer Internet-Kundenberatung}

Die Verknüpfung bestehender Dienstleistungsangebote einer Bank mit einer audio-visuellen Kundenberatung im Internet stellt eine absolute Neuheit dar und bringt somit für den Anbieter eine Vorreiterrolle und einen Imagevorsprung mit sich.

IDS Prof. Scheer GmbH hat hierzu einen Prototypen entwickelt, der eine audiovisuelle Internet-Beratung ermöglicht. Anhand der (fiktiven) Y-Bank wird dargestellt, wie die individuelle Kundenberatung in das bisherige Online-Angebot einer Bank integriert werden kann.

Das Beratungsangebot ergänzt das bislang bestehende Angebot des Finanzdienstleisters durch die Möglichkeit der direkten Kontaktaufnahme des Kunden mit dem Kundenberater in der Bank.

Die Kundenberatung über das Internet stellt sich als ein Prozeß dar, der vom Kunden initiiert wird. Anlaß wird häufig eine Frage des Kunden zum bestehenden Internet-Angebot oder darüber hinaus sein. Beantwortet werden die Fragen der Kunden im Call-Center des Finanzdienstleister, wobei die Möglichkeit besteht, einen bestimmten Kundenberater anzuwählen. Im Dialog mit dem Kunden besteht dann für den Kundenberater die Möglichkeit einer umfassenden Finanzberatung, die den individuellen Bedürfnissen des Kunden 
entspricht. Dabei können nicht nur verbale Erläuterungen geliefert, sondern auch Beispielrechnungen oder Charts erstellt und präsentiert werden.

Für Bank- und Versicherungskunden ist dieses Service-Angebot attraktiv, zumal sie keine Zusatzaufwendungen haben. Die Voraussetzungen für die Nutzung der audio-visuellen Internet-Beratung werden von nahezu allen Internet-Usern erfüllt. Erforderlich sind ein PC mit Internet-Zugang, Soundkarte, Lautsprecher oder Kopfhörer und Mikrophon.

Sofern eine Bank oder Versicherung über ein Call-Center verfügt oder dessen Aufbau plant, ist die audio-visuelle Beratung auch für den Anbieter mit minimalen Investionen verbunden. Die Computer an den Call-Center-Arbeitsplätzen müssen lediglich mit einer IP-Verbindung zum Internet, mit Sound- und Videokarte bzw. Videokamera sowie Mikrophon ausgerüstet werden.

Aufgrund der Öffentlichkeit, die durch ein so innovatives Angebot erreicht wird, ist es aber für viele Finanzdienstleister empfehlenswert, zeitgleich oder vorab ihre Internet-Seiten "web-to-date" zu gestalten (Damaschke 1998). Vor allem aber ist dafür zu sorgen, daß die organisatorischen Voraussetzungen pünktlich zum Online-Start geschaffen sind, so daß ein Nutzer keine negativen Überraschungen erlebt und der erwünschte Image-Gewinn ausbleibt.

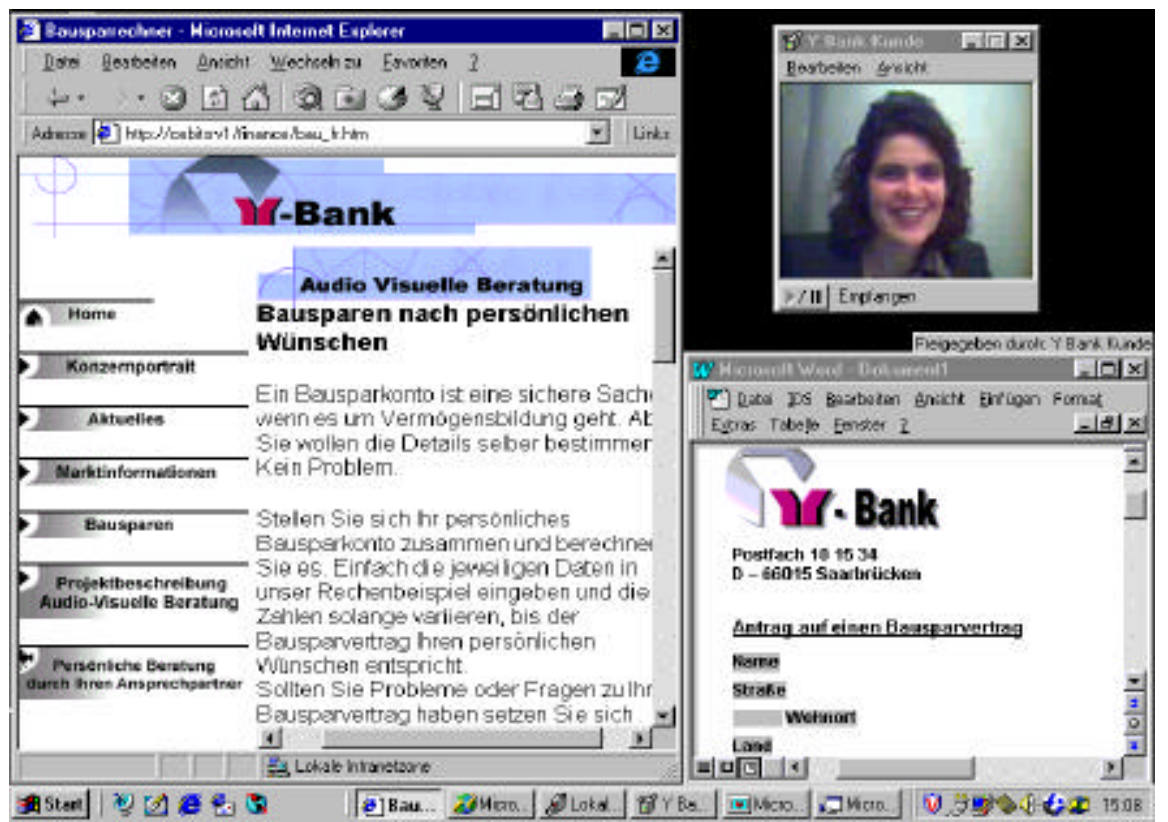

Abbildung 2: Beispiel einer Beraterbank mit Integration von WWW, Videokonferenz und Shared Applications 
Organisatorische Voraussetzungen der Internet-Beratung sind insbesondere die Gewährleistung der Erreichbarkeit sowie die Gewährleistung einer kompetenten, freundlichen, verkaufsorientierten und dem technischen Medium angepaßten Beratung. Die Anpassung an das technische Medium erfordert eine Schulung der eingesetzten Call-Center-Mitarbeiter. Die Tatsache, daß der Call-Center-Mitarbeiter sichtbar ist und somit nicht nur mit seiner Stimme, sondern auch mit seinem Aussehen und seiner Mimik das Unternehmen repräsentiert muß beachtet werden.

Im ersten Schritt sollte die Internet-Beratung an einer geschlossenen Benutzergruppe erprobt werden. In Frage kommen hier z. B. Außendienstmitarbeiter eines Finanzdienstleisters (Rahlenbeck 1997, S. 30) oder eine ausgewählte, für den Finanzdienstleister besonders wichtige Kundengruppe, der ein besonderer Service offeriert werden soll. Hierbei sollten auch die angebotenen Produkte auf die Zielgruppe und das Medium Internet zugeschnitten sein. Ein Pilotprojekt mit einer großen Zielgruppe sollte nicht mit den beratungsintensivsten Produkten begonnen werden.

Der erste Schritt in Richtung eines solchen Konzeptes ist bereits von der Advance Bank umgesetzt worden. Diese ermöglicht es Kunden, sich via Internet ein auf die persönlichen Bedürfnisse zugeschnittenes Baufinanzierungsangebot errechnen zu lassen, welches anschließend gemeinsam mit einem Online-Berater der Bank diskutiert bzw. modifiziert werden kann (Buhl et al. 1998, S.78 f.).

\section{Module einer interaktiven Internet-Beratung}

Bei der Umsetzung von persönlichen Beratungsleistungen im Internet können drei verschiedene Module entweder simultan oder zeitlich versetzt zur Kommunikation zwischen Kunde und Bank genutzt werden. Dies sind Audio-, Video- und Daten-Konferenz-Modul.

Diese Module werden über eine E-Services-Plattform zu einer flexiblen und sicheren Lösung zusammengeführt. Eine solche Lösung muß die sichere Übertragung von vertraulichen Daten gewährleisten, einfach erweiterbar sein, internationale Telekommunikationsstandards unterstützen, leicht in eine bestehende IT-Infrastruktur integrierbar sein und die Ladezeiten beim Kunden minimieren.

In den folgenden Unterpunkten werden im Anschluß die einzelnen Module einer interaktiven Internet-Beratung mit Schwerpunkt Audio-Video-Beratung näher betrachtet. 


\subsection{Daten-Konferenz-Modul}

Die Implementierung von Daten-Konferenz-Funktionalitäten in das Beratungskonzept bietet viele Möglichkeiten, einen direkten, persönlichen Kontakt zum Kunden aufzubauen. In einem Finanz-Beratungs-Szenario können die Funktionen Chat, Whiteboarding und Application Sharing implementiert werden.

\subsubsection{Chat Systeme}

Schon seit es Telekonferenzen auf Mainframe Systemen und Minicomputern gibt, ist es möglich, via Chat kurze Mitteilungen auszutauschen, die in einem gemeinsamen Konferenzfenster angezeigt werden. Mittels eines Start-ChatButton kann ein Chat Fenster aufgerufen werden. Jeder Teilnehmer hat eine lokale Message Box, um Nachrichten zu schreiben und zu bearbeiten, sowie ein Menü mit einer Send-To Liste. Jede empfangene Mitteilung wird in einem gemeinsamen Chat-Fenster zusammen mit dem Namen des Senders angezeigt.

Diese Funktion eignet sich im Rahmen eines Beratungsszenario für die schnelle und unkomplizierte Beantwortung von wenig komplexen Fragen. Beispielsweise können Informationen über Konditionen und Termine auf diesem Weg übertragen werden.

\subsubsection{Whiteboard}

Das sogenannte Whiteboarding ist eine wesentlich effektivere Methode, um Informationen auszutauschen. Jeder Teilnehmer sieht hierbei eine Art Notizblock. Texte oder Bitmap-Bilder, die in den Notizblock kopiert werden, werden auf den Notizblöcken aller Teilnehmer sichtbar.

Die Fähigkeit, Bilder auszutauschen, ist im T.120 (Transmission Protocols for Multimedia Data) Standard definiert. Bis heute entsprechen allerdings nur wenige Produkte voll dem Standard, was eine Kommunikation zwischen den Produkten verschiedener Hersteller oder unterschiedlicher Programmversionen sehr schwierig macht.

Eine Implementierung dieser Funktionalität ermöglicht die Beantwortung komplexerer Fragen, da hier Grafiken eine wesentliche Hilfestellung bieten. Solche Grafiken können bei der Bank für die einzelnen Fragestellungen schon im voraus erstellt werden, um so eine schnelle Beantwortung der Anfragen zu ermöglichen.

\subsubsection{Application Sharing}

Beim Application Sharing geht es darum, daß zwei oder mehrere Benutzer von unterschiedlichen Lokationen aus gemeinsam eine Datei bearbeiten können. Grundsätzlich verarbeiten diese Programme Bildschirm-, Maus- und TastaturEvents von verschiedenen Teilnehmern. Dabei wird zunächst eine Netzwerk- 
verbindung zu einem Host-PC aufgebaut, und im Anschluß erscheint ein Fenster mit dem Bildschirminhalt des Host-PCs. Maus und Tastatur sind bei beiden Teilnehmern aktiv, so daß jeder Dateien öffnen und bearbeiten kann.

Die Möglichkeit, gleichzeitig Banking-Applets $\mathrm{zu}$ bearbeiten und ein Application-Sharing durchzuführen, ist stets mit der Freigabe vertraulicher Daten verbunden. Hier ist der Einsatz von Java-Applets zu bevorzugen, die genau auf die vom Anbieter eingesetzten Banking-Applikationen zugeschnitten sind und nur ein Sharing genau definierter Bereiche bzw. Daten ermöglichen. Je transparenter dies geschieht, desto höher wird auch das Vertrauen des Kunden in die Sicherheit des Systems ausfallen.

Eine speziell auf diese Anforderungen zugeschnittene Lösung ermöglicht ein gemeinsames Bearbeiten einer Finanztransaktion, ein gemeinsames Browsing, das "Pushen" von URLs und die Möglichkeit, mit Hilfe einer Zeichenfunktion im Browser-Fenster des Kunden Markierungen vorzunehmen. Auf diese Weise ist es beispielsweise dem Call-Center-Mitarbeiter möglich, gemeinsam einen Kaufauftrag für Aktien auszufüllen, den Kunden gezielt durch spezielle Web-Seiten zu navigieren oder ihn auf wichtige Informationen zu verweisen.

\subsection{Audiomodul}

Für die Umsetzung der Audiokommunikation gibt es grundsätzlich zwei Möglichkeiten: Zum einen die Kommunikation über das traditionelle Telefonnetz, zum anderen den Verbindungsaufbau über das Internet.

Der Nachteil der ersten Variante ist, daß es zu einem Medienbruch kommt und der Kunde - falls er nicht über einen freien Telefonanschluß verfügt (z. B. Modem blockiert den einzigen Anschluß) - diese Interaktion mit dem Call-Center nicht simultan zu seiner Internet-Session in Anspruch nehmen kann. Auf Grund des Medienbruchs ist es selbst bei einer simultanen Inanspruchnahme von Internet und Telefon kaum möglich, eine Synchronisation zwischen der Audioübertragung und einer möglichen Videoübertragung zu erreichen.

Allerdings besteht der Vorteil eines solchen Aufbaus in der garantiert hohen Übertragungsqualität und der hohen Verfügbarkeit, die in diesem Maß via Internet-Telefonie noch nicht sichergestellt werden kann.

Um eine Anpassung an künftige Entwicklungen zu vereinfachen und um innovativen Kunden zusätzliche Möglichkeiten zu bieten, sollte von Beginn an auch die Möglichkeit gegeben sein, den Audiokanal via Internet aufzubauen.

Die Vorteile eines solchen Angebots liegen darin, daß es keinen Medienbruch mehr gibt und es dadurch einfacher wird, den Audiokanal simultan zu den anderen Kanälen zu nutzen. 


\subsection{Videomodul}

Für den Aufbau von Live-Videokonferenzen via Internet gibt es den ITU Standard H.323, welcher die Interoperabilität zwischen Systemen verschiedener Hersteller gewährleistet. Die Herausforderung beim Aufbau einer LiveVideoverbindung stellt der enorme Bandbreitenverbrauch dar. Letztendlich entscheidend für die Akzeptanz einer solchen Videokommunikation ist, daß die Technologie vielseitig (mit annähernd jedem Kamera- oder Soundsystem auf einem PC funktionierend), flexibel (ermöglicht Konferenzen zwischen mehreren Teilnehmern, gleichgültig ob deren Terminals video- oder audiofähig sind), robust und intelligent (ermöglicht Verbindungen auf Grund von ILS (Internet Locator Service), der Emailadresse oder der IP-Adresse) ist. Damit kann trotz sehr unterschiedlicher technischer Voraussetzungen bei verschiedenen Kunden ein qualitativ hochwertiger Service bereitgestellt werden. Der Aufbau einer LiveVideokonferenz stellt derzeit nicht den Focus der Beraterbank im Internet dar. Dies wird sich jedoch sicherlich im Lauf der Zeit ändern, sobald die erforderlichen Bandbreiten zur Verfügung stehen und Dienstleistungen vom Finanzinstitut angeboten werden, die dies erfordern.

Derzeit sieht das Konzept der Beraterbank im Internet vielmehr eine einseitige Übertragung von Videosequenzen von der Bank zum Kunden vor. Dies hat auch zur Folge, daß die Hardwareausstattung beim Kunden wesentlich geringer ausfallen kann als für "echtes" Videoconferencing. Vorstellbar ist ein Szenario, bei dem die Begrüßung des Kunden per Videosequenz erfolgt, anschließend jedoch durch ein Standbild ersetzt wird. Sofern über eine Mindestbandbreite zur Datenübertragung verfügt werden kann, können auch während der weiteren Beratung Bewegtbilder übertragen werden. Da im Rahmen der Videokommunikation keine vertraulichen Daten übertragen werden, gibt es hierbei auch keine speziellen Sicherheitsanforderungen.

Der visuelle Kontakt zum Berater stellt vor allem für den Aufbau von Vertrauen einen nicht zu unterschätzenden Faktor dar. Der Kunde bekommt das Gefühl, tatsächlich mit einem Menschen zu kommunizieren und das Medium Internet erfährt dadurch eine gewisse Humanisierung.

\subsection{Call-Me Button}

Konkret wird die Audiokommunikation durch Implementierung eines Call-Me Buttons in die Web-Seiten bzw. die Java-Applikation umgesetzt. Der Kunde kann hierbei durch Klicken auf diesen Button sofort eine Audioverbindung zum Bankberater aufbauen, der ihm sozusagen auf "Knopfdruck" mit Rat und Tat zur Verfügung steht. Dieses Beratungsangebot soll unterstützend und ergänzend wirken. 


\section{Aufbau einer sicheren und flexiblen Lösung}

Um die angesprochenen Module zu einer Gesamtlösung zusammenzuführen und diese sicher und einfach mit der bestehenden IT-Infrastruktur zu verknüpfen, stellt eine E-Services-Plattform die ideale Lösung dar. Die Plattform gewährleistet die sichere Einbindung eines für die Umsetzung einer persönlichen Beratung wichtigen Internet-Call-Centers. Im folgenden wird der Aufbau einer sicheren und flexiblen Beratungslösung mit den Komponenten E-ServicesPlattform und Internet-Call-Center beschrieben.

\subsection{E-Services-Plattform als zentrales Element}

Wesentlich für eine erfolgreiche Umsetzung eines Beratungskonzeptes ist die Anbindung aller Module an die bestehende IT-Infrastruktur. Das Angebot professioneller, elektronischer Services erfordert ein Maximum an Flexibilität und Zuverlässigkeit der eingesetzten IT-Systeme.

Eine State-of-the-Art-Lösung für Electronic Services Delivery ermöglicht es, auf Basis einer einzigen, integrierten Plattform mehrere elektronische Distributionskanäle zu erschließen, deren Funktionalitäten kanalübergreifend verknüpft werden können, während gleichzeitig eine volle Integration mit beliebig heterogenen Backend-Systemen möglich ist.

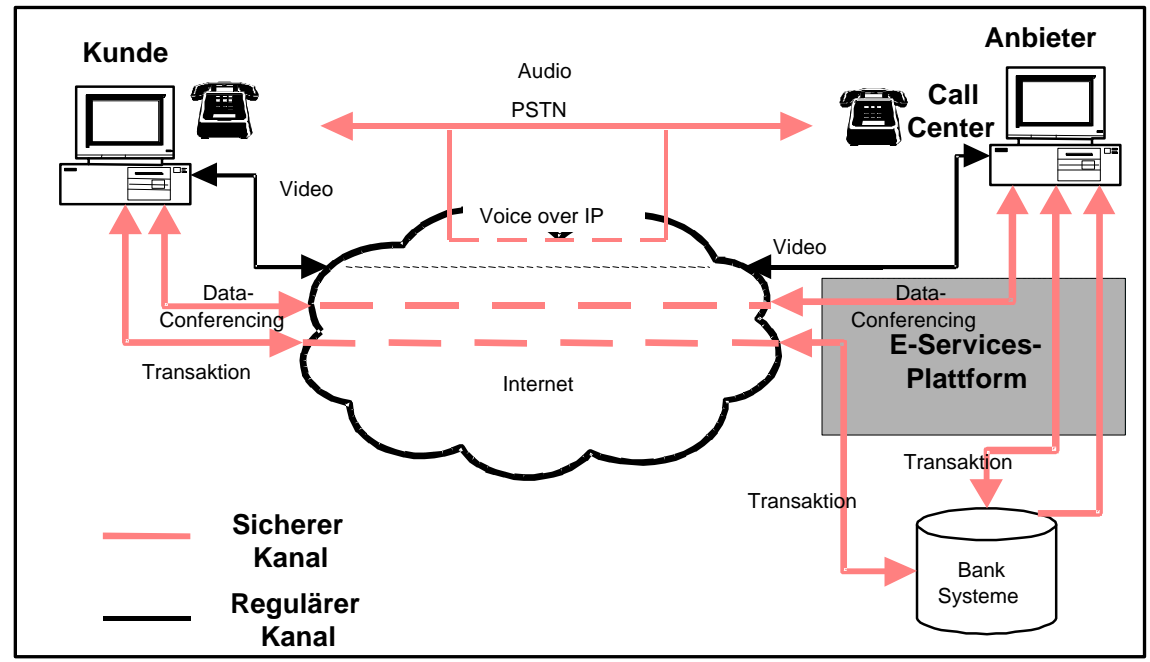

Abbildung 3: Beispiel einer Beraterbankarchitektur

Eine E-Services-Plattform stellt Komponenten bereit, die schnell und unkompliziert Zugang zu Electronic Delivery Channels wie Internet oder Mobilfunknetzen schaffen und gleichzeitig eine einfache Einbindung bestehender IT- 
Umfelder in die dort angebotenen Module erlauben. Eine E-Services-Plattform sollte neben einer Gewährleistung von Offenheit durch die Unterstützung von Industriestandards wie CORBA oder COM ein integriertes Sicherheitskonzept bieten, das unternehmenskritischen Anwendungen Mechanismen zur Gewährleistung von Authentizität, Integrität, Vertraulichkeit und Nicht-Abstreitbarkeit aller abgewickelten Vorgänge zur Verfügung stellt.

\subsubsection{Sicherheitskonzept}

Entscheidend für eine erfolgreiche Implementierung von Beratungs-Services ist die Gewährleistung der Sicherheit. Da in einer Beratungssituation unter Umständen vertrauliche Daten übertragen werden, muß hier mindestens ein ebenso hoher Sicherheitsstandard gewährleistet sein wie bei der Abwicklung von Finanztransaktionen. Von zentraler Bedeutung zur Gewährleistung der sicheren Übertragung im offenen Internet ist der Nachweis der folgenden Eigenschaften: Vertraulichkeit, Integrität und Authentizität.

\section{Vertraulichkeit}

Die Vertraulichkeit wird durch Verschlüsselung der Nachrichten erreicht. Moderne Verschlüsselungssoftware kann Nachrichten automatisch ver- und entschlüsseln. (Schmeh 1997) Mit dem SRT-Protokoll (Secure Request Technology) wird eine leistungsoptimierte Variante des bekannten NetscapeProtokolls SSL (Secure Sockets Layer) implementiert. Für "Hacker" stellen die eingesetzten Verschlüsselungsalgorithmen RSA-1024 Bit und IDEA-128 Bit ein unüberwindliches Hindernis dar. Die Verschlüsselungs-Kommunikation über das SRT-Protokoll wird auf der Request- oder Anwendungsebene durchgeführt. Dadurch ergibt sich ein besonderer Vorteil: Eine SRT-Verschlüsselung kann zusätzlich zu existierenden Browser-Verschlüsselungen durchgeführt werden. So kann eine noch höhere Transaktionssicherheit (40 plus 128 Bit) erzielt werden. Transaktionen werden in beide Richtungen mit hoher Leistung ver- und entschlüsselt. Dazu werden symmetrische Sitzungsschlüssel (session keys) verwendet, die automatisch zwischen Client und Server ausgehandelt werden (Röver, Schlumpberger 1996).

\section{Integrität}

Die Integrität einer Transaktion bedeutet, daß die Nachricht während der Übertragung nicht unbemerkt verändert wurde. Hierzu verwendet man den Vergleich von elektronischen Fingerabdrücken vor und nach der Übertragung. Als elektronischer Fingerabdruck wird ein sogenannter MAC (Message Authentication Code) eingesetzt. Ein MAC wird durch eine sichere EinwegFunktion erzeugt, die auf die gesamte Nachricht angewendet wird. Jeweils vor und nach einer Datenübertragung werden MACs unter Verwendung der entschlüsselten Nachricht berechnet. Ein Vergleich beider MACs zeigt an, ob die Nachricht manipuliert wurde. 


\section{Authentizität}

Die Authentifizierung der Kommunikationspartner kann durch unterschiedliche Verfahren, je nach gefordertem Sicherheitslevel umgesetzt werden.

Möglich ist hier der Einsatz von PIN/TAN Verfahren, Digitalen Signaturen, wobei der private Schlüssel auf dem PC oder auf einer Smart Card gespeichert wird oder der Einsatz von sogenannten Challenge/Response Tokens (Anderer 1998).

Eine indirekte Authentifizierung des Servers kann erreicht werden, wenn die Transaktions-Applets, die den öffentlichen Schlüssel des Security Servers enthalten, über einen zertifizierten SSL Protokollstrom geladen werden. Ein solcher Protokollstrom wird unter Verwendung des öffentlichen RSA Schlüssels eines SSL Web-Servers aufgebaut, der z. B. von der Firma VeriSign, USA, zertifiziert ist. Verisign-Zertifikate für Server und Browser sind ein weltweiter Defacto-Standard. Voraussetzung ist die Verwendung eines Java-Browsers und Web-Servers, die Zertifikate und SSL unterstützen. Nahezu alle führenden Web-Server- und Browserfirmen, die SSL implementiert haben, unterstützen VeriSign-Zertifikate. Ein öffentlicher RSA Schlüssel mit VeriSign-Zertifikat kann eindeutig dem Ursprungs-Web-Server zugeordnet werden, d. h. daß die Applets im Client Browser nur mit dem Ursprungs-Web-Server kommunizieren.

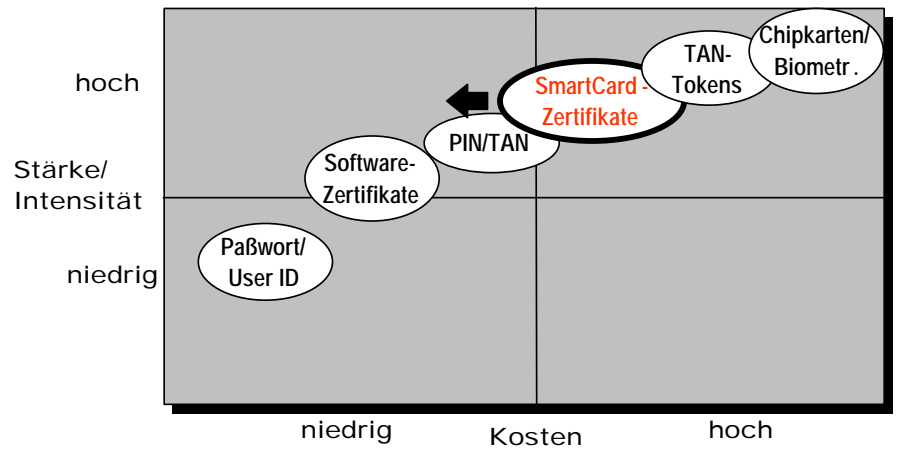

Abbildung 4: Authentifizierungs-Methoden

\section{Firewall}

Firewall-Systeme stellen spezielle Sicherheitssysteme dar, um Firmendaten sowie das interne Netz vor unerwünschten Zugriffen zu schützen. Mit dem FirewallSystem läßt sich die Kommunikation zwischen dem Internet und bankinternen Netzwerken kontrollieren. Daher hat die Verhinderung von Einbrüchen in das Bank-Netz einen wesentlichen Anteil am gesamten Sicherheitskonzept. 


\subsubsection{Sicherheitskonzept in der Firewall-Peripherie}

Grundsätzlich gilt in einem sicheren System, daß alles nicht explizit Erlaubte verboten ist. Um das Gesamtsystem sicher zu machen, sollten folgende Punkte beachtet werden:

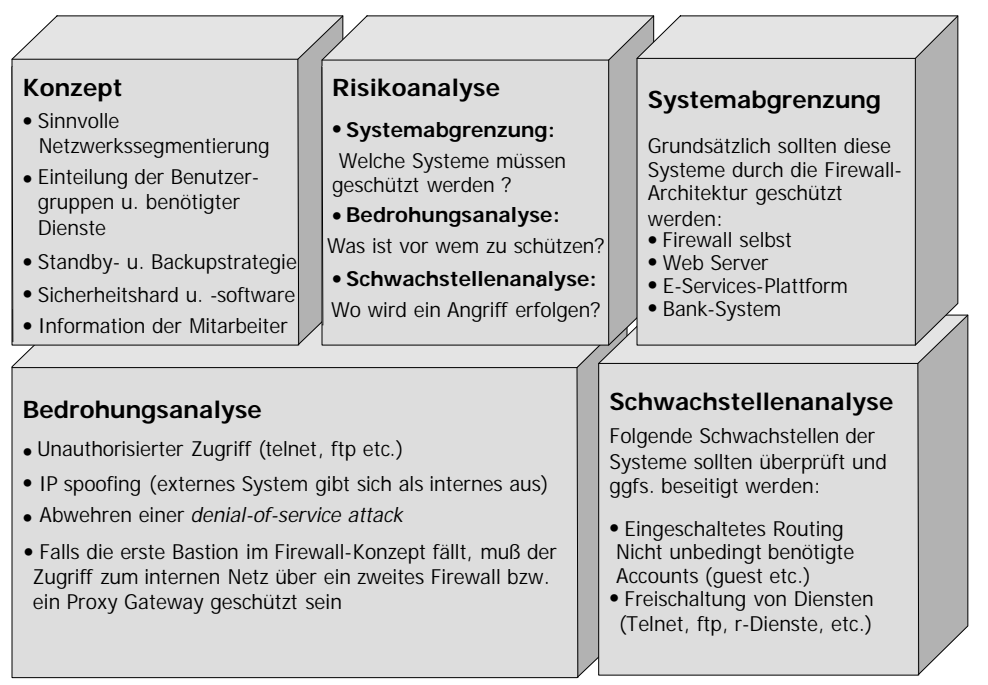

Abbildung 5: Sicherheitskonzept in der Firewall Peripherie

\subsubsection{Klassische Firewall-Konzepte}

Paket-Filter Router stellen die einfachste Methode dar, um eine Direktverbindung zum Internet einzurichten. Diese Paketfilter betrachten die Header Informationen (Quell- und Zieladresse sowie IP-Port). Viele der Router-Hersteller erlauben Regeln zum Filtern, die den Eingang und den Ausgang der verschiedenen Pakettypen (z. B. Telnet) definieren. In der Vergangenheit konnten einige Router die Zugriffsregeln umorganisieren, um einen Leistungszuwachs zu erzielen. Das war und ist natürlich ein Alptraum für den Administrator, der gerade sichere Zugriffsregeln einrichten will. Praktisch alle Hersteller haben aber inzwischen diesen Mangel abgestellt.

Ein ungelöstes Problem bei diesem Gateway-Typ ist aber der Umstand, daß sich das Unternehmen auf die Sicherheitsmaßnahmen verlassen muß, die in der Anwendung eingebaut sind. Wohl eines der bekanntesten Beispiele für dieses Problem ist ein fehlerhaft konfigurierter "Sendmail". (Der berühmte InternetWurm reiste auf dem STMP-Protokoll, und wenn der Filter Router-Zugriff auf eingehende E-Mail erlaubte, konnte der Wurm in das Unternehmensnetz gelangen. Da er die Schwächen des Sendmail-Programms ausnützte - nicht des STMPProtokolls - konnte er so die privaten Hosts befallen und lahmlegen.) 
Application oder Proxy Gateways werden für Telnet, ftp und andere Dienste genutzt, die eine Verbindung zum Internet benötigen. Sie werden an Stelle von direkten Verbindungen zum Internet verwendet, um einerseits guten Zugriff zu den Diensten auf dem Netz zu erlauben und andererseits soviel Sicherheit wie möglich zu gewährleisten. Die Anwender in privaten Netzwerken erhalten dabei keine direkte Verbindung zum Internet. Vielmehr wird vom privaten Netzwerk eine Verbindung zum Application Gateway auf dem Firewall-Host aufgebaut. Das Gateway gibt dann die Anforderung an den entsprechenden Bestimmungsort im Netz weiter. Die Anwender können dabei im Netz arbeiten, als wären sie direkt mit dem Host im Internet verbunden.

Application Gateways verfügen auch über zusätzliche Sicherheits-Prüfungen. Das Gateway untersucht jede Verbindung bis ins tiefste Detail nach folgenden Kriterien:

- Host, von dem die Kommunikations-Verbindung angefordert wurde

- Host, zu dem die Kommunikations-Verbindung aufgebaut wird

- Befehle, die über die Verbindung eingegeben werden.

Dies erlaubt dem Administrator, das Gateway so zu konfigurieren, daß alle Verbindungen zu spezifizierten Routern, Netzwerken und/oder Rechnern im Internet ausgeschlossen werden, d. h. das private Netz sehr sicher gehalten werden kann. Über das Gateway kann auch verhindert werden, daß Anwender Befehle eingeben, die ein potentielles Risiko beinhalten (z. B. Befehle, die Informationen aus dem privaten Netzwerk ins Internet exportieren können).

Einer der Nachteile des Application Gateways ist, daß einige Netzwerk-Anwendungen einen zweistufigen Prozeß zum Verbindungsaufbau benötigen. Es wird ein Sprung zur internen Verbindung und dann zur externen (bzw. umgekehrt) benötigt.

\subsubsection{Sicherheits-Architektur}

Die Vorteile der Paketfilter liegen in der Transparenz und in der Performance. Proxy Gateways bieten hingegen ein hohes Maß an Sicherheit bei verminderter Performance. Um die Vorteile beider Systeme zu nutzen, sollte das Firewall Konzept des Internet On-Line Systems das jeweilig beste System in kaskadierter Anordnung benutzen.

Hierzu sollten der Web- und der Security-Server in einer DMZ (Demilitarisierten Zone) angeordnet werden. Die DMZ wird in Richtung des Internets durch ein Firewall mit Paketfilter (Nur http und SSL Datenströme) sowie IP Adress Translator (Nur eine IP Adresse zum Internet, die mit der IP Adresse des WebServers verbunden ist) geschützt. Dies ermöglicht dem Kunden, den Web-Server mit hoher Performance anzusprechen. Weitere Zugriffsmöglichkeiten vom Internet in die DMZ sind nicht vorhanden. Der Web Server kommuniziert über CGI Requests mit dem, meist auf dem gleichen System eingesetzten, Security Server. 
Wird dieses System mit zwei Netzwerkadaptern als Dual Homed Gateway konfiguriert (kein Routing), bildet es ein Proxy Gateway.

Der Security Server kommuniziert über ein zweites Firewall mit der E-ServicesPlattform. Die zweite Firewall Bastion beinhaltet einen Paketfilter, der nur die Verbindung zwischen der IP-Adresse des Security Servers und der IP-Adresse der E-Services-Plattform auf einem bestimmten Port zuläßt.

Als vierte Bastion wird die E-Services-Plattform als Proxy Gateway eingesetzt. Durch den Einsatz eines Firewall-Systems mit vier Netzwerkadaptern wird trotz vierstufigem Firewallkonzept nur eine zusätzliche Hardwareplattform sowie eine Firewall-Lizenz benötigt.

Ein weiterer Sicherheitsvorteil der DMZ liegt in der Möglichkeit, bei Wartungsarbeiten an den Systemen in der DMZ das Firewall 1 zum Internet hin vollkommen abzuschotten. Daraufhin kann das Firewall 2 für Zugriffe aus dem internen Banknetz freigeschaltet werden.

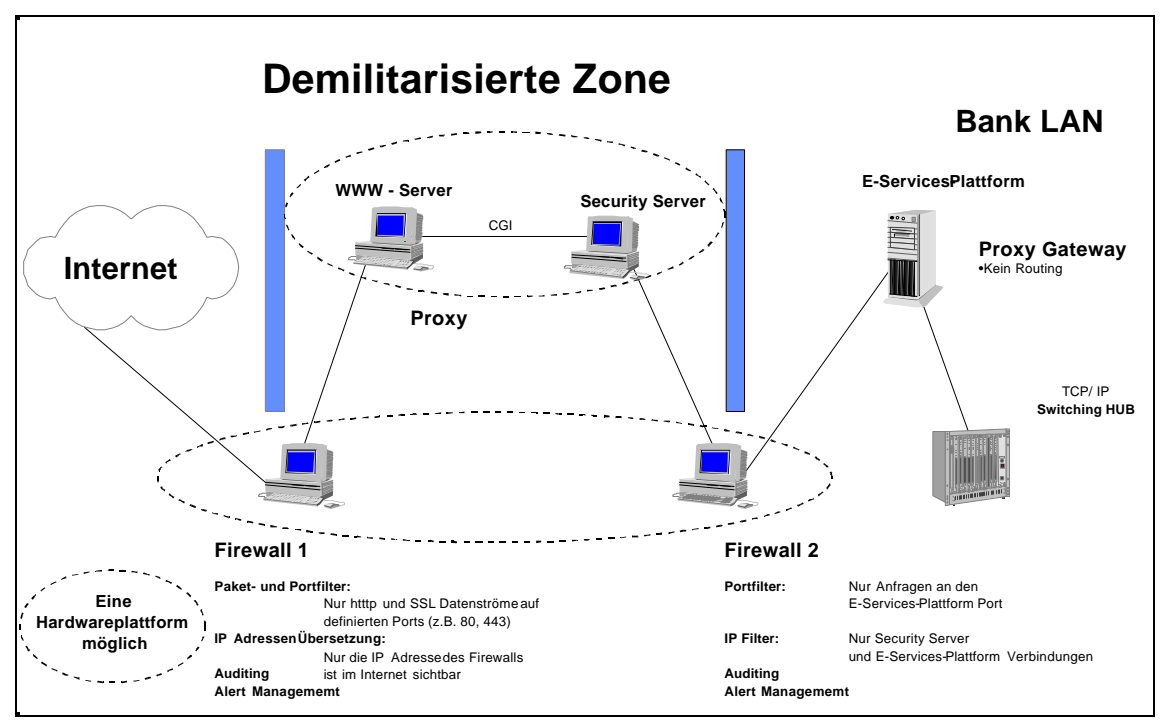

Abbildung 6: Sicherheits-Architektur

\subsection{Architektur eines Internet-Call-Centers}

Mit Hilfe der E-Services-Plattform wird ermöglicht, daß die einzelnen Beratungsmodule sicher und einfach an ein Internet-Call-Center angebunden werden können. Dabei wird gewährleistet, daß das Bank-Netz vor unauthorisierten $\mathrm{Zu}$ griffen geschützt wird. Die Call-Center-Software gewährleistet, daß Anfragen des Kunden schnell an die zuständigen Agenten weitergeleitet werden. Es wird 
außerdem gewährleistet, daß der Kunde genau mit den von ihm ausgewählten Kommunikationsmodulen bedient wird.

Call-Center-Software ist normalerweise keine Standardsoftware und wird für die jeweilige Lösung angepaßt (programmiert). State-of-the-Art sind GUI-Anwendungen auf dem jeweilig eingesetzten System (Unix /Windows). Zunehmend setzt sich Computer Telephony Integration (CTI) durch. Unter CTI versteht man die Zusammenführung von Sprach- und Datenkommunikation. Dadurch wird es möglich, dem Telefonisten (Agent) zusammen mit dem Telefongespräch die Kundendaten zu übermitteln. Außerdem ermöglicht CTI ein komplexes CallRouting, die Übermittlung von Sprache und Daten über eine Leitung und die Integration von Internet (e-mail) und Video (Bildtelefonie).

Bei den angeschlossenen Back-End Systemen handelt es sich meistens um Datenbankapplikationen (Kundenstamm-Verwaltung).

\section{Generischer Call-Center-Aufbau}

Call-Center können weitestgehend modular aufgebaut werden. Entscheidend für die Größe des Call-Centers sind die zu erwartende Anruferzahl pro Zeitabschnitt (Std.) bzw. die Anzahl benötigter Telefonisten (Agenten bei aktivem Telefonmarketing).

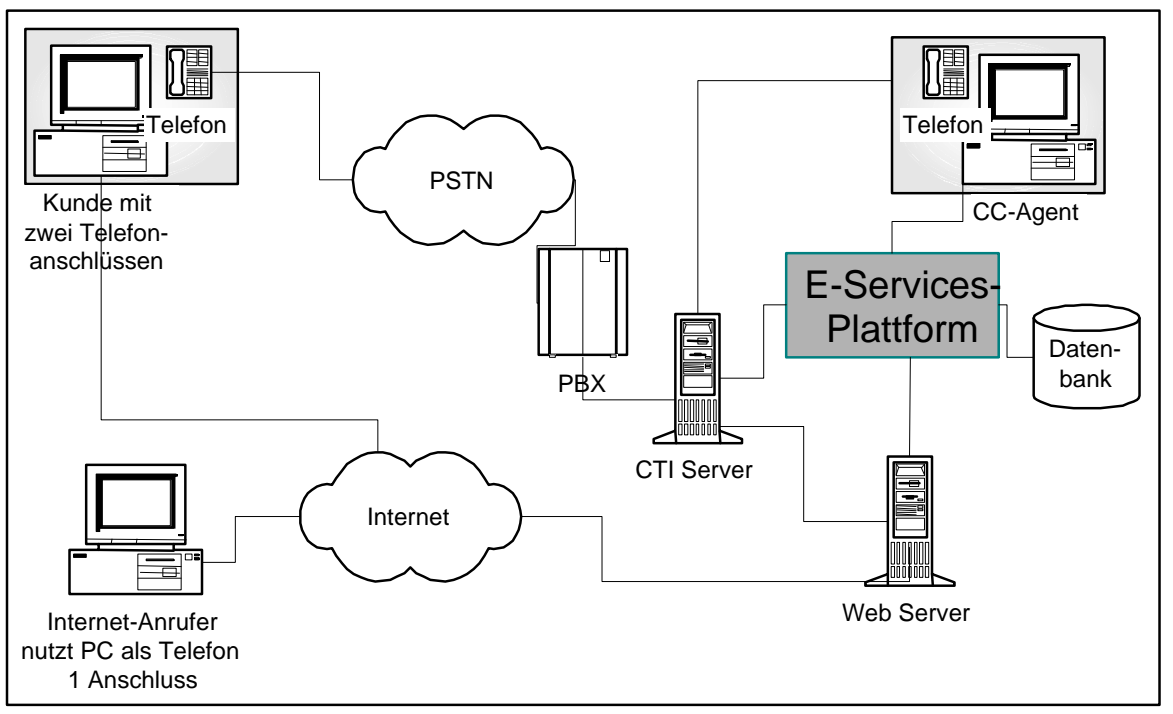

Abbildung 7: Call-Center-Aufbau

Call-Center können weitestgehend modular aufgebaut werden. Entscheidend für die Größe des Call-Centers sind die zu erwartende Anruferzahl pro Zeitabschnitt (Std.) bzw. die Anzahl benötigter Telefonisten (Agenten bei aktivem Telefonmarketing). 
Oftmals werden einzelne Call-Center-Komponenten redundant ausgelegt, um eine höchstmögliche Verfügbarkeit zu garantieren.

Ein modernes Call-Center besitzt mindestens eine Privat Branch Exchange (PBX): Eine Telefonanlage (Nebenstelle), welche die Verbindung zum Telefonnetz herstellt. Hier werden die eingehenden Gespräche entgegengenommen. Die PBX verteilt Anrufe an angeschlossene Komponenten (Automated Call Distribution), z. B. bei verteilten Call-Centern. Über Dialed-Number-Identification-Services oder Automatic-Number-Identification-Services können zusätzliche Informationen gesammelt und für Intelligent-Routing genutzt werden.

Weitergeleitet werden die Anrufe von der PBX an eine CTI-Komponente. CTIKomponenten sind die Hauptbestandteile moderener Call-Center. Je nach Ausstattung bieten sie Interactive-Voice-Responce, Skill-based Routing, QueueManagement und Reporting Funktionen. Zudem ist es mit CTI möglich, den Anruf weiterzuleiten und gleichzeitig auf dem Bildschirm des Agenten die Kundendaten anzuzeigen. Zu diesem Zweck besitzt die CTI-Komponente Schnittstellen für den Zugriff auf Datenbanken bzw. Host-Systeme.

\section{Beraterbank-Paket}

Eine erste Entwicklungsstufe eines Beraterbank-Paketes stellt die Implementierung eines sicheren 1TO1-Chatsystems und die Möglichkeit der gemeinsamen Bedienung des Browsers dar, welche mit Hilfe von Java-Applets realisiert wird. Außerdem werden an die verfügbaren Bandbreiten angepaßte Videoangebote bereitgestellt und die Möglichkeit eines Rückrufs über PSTN oder Internet angeboten. Als weitere Schritte können dann sowohl Whiteboarding als auch "echtes" Application Sharing umgesetzt werden. 


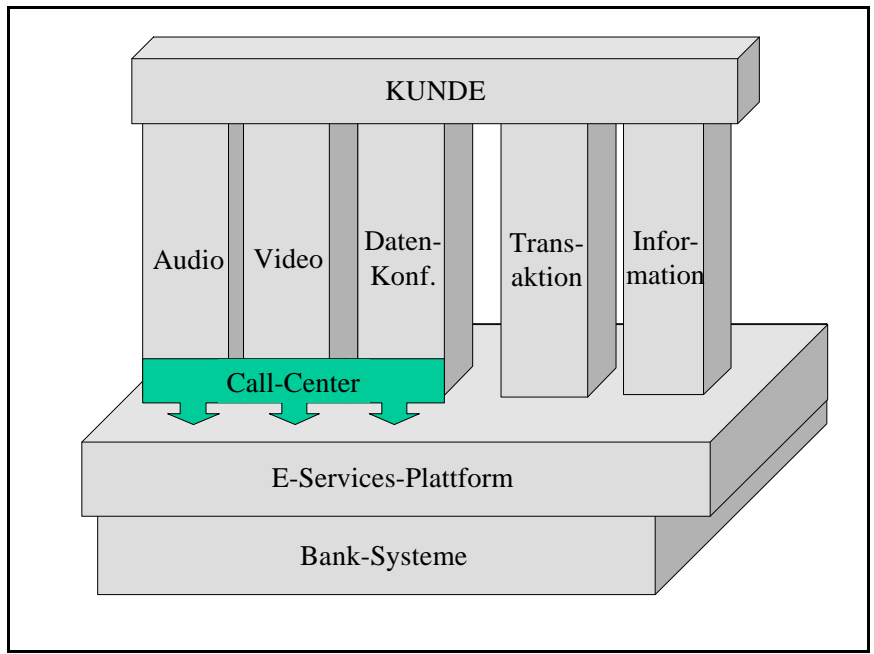

Abbildung 8: Beraterbank-Paket

Daraus resultiert ein modular aufgebautes Beraterbank-Paket, daß es dem Anbieter ermöglicht, je nach Beratungssituation unterschiedliche Beratungsmodule einzusetzen. Dabei werden ebenfalls schon bestehende Transaktions- und Informationsmodule mit der Beratungslösung verbunden, um so für den Kunden ein konsistentes Bild zu schaffen. Durch Unterstützung von Industriestandards bildet die E-Services-Plattform - über welche die Kommunikation zwischen Kunde und Anbieter abläuft - den Schlüssel, um einen solchen Aufbau zu realisieren. Ein modularer Aufbau hat außerdem den Vorteil, daß eine bereits implementierte Lösung jederzeit um weitere Module erweitert werden kann.

Es sollte stets beachtet werden, daß ein wesentlicher Vorteil der Kommunikation mit dem Kunden via Internet darin besteht, daß ein Großteil der Fragen des Kunden automatisch beantwortet werden kann. Die Implementierung von Beratungsleistungen sollte daher zweistufig vorgenommen werden. Der Kunde bekommt dann bei Fragen, für die automatisch keine Antwort generiert werden kann, die Möglichkeit, das interaktive, persönliche Beratungsangebot in Anspruch zu nehmen.

Um diesen persönlichen Kontakt zu realisieren, spielt die Einbindung eines Internet-Call-Centers eine entscheidende Rolle. Die Yankee Group spricht in diesem Zusammenhang sogar von Cyber Call-Centern (The Yankee Group, "Cyber CallCenters 1998: From Internet Enabled to Internet Architected Call-Centers,"). Die sichere und einfache Anbindung des Call-Centers erfolgt mit Hilfe einer EServices-Plattform. Die Verbindung der interaktiven Beratung mit den Möglichkeiten, gleichzeitig Transaktionen durchzuführen oder Informationen abzurufen, stellt für den Kunden einen entscheidenden Nutzen-Zuwachs dar und sorgt so für eine stärkere Kundenbindung. 
Eine erste Implementierung des Beraterbank-Paketes kann, anstatt direkt beim Kunden, auch mit Hilfe eines Web-Kiosks in ausgewählten Filialen oder BankShops erfolgen. Dadurch bietet sich, auf Grund der größeren Bandbreiten, die Möglichkeit, dem Kunden eine größere Funktionalität zur Verfügung zu stellen und ihn mit einem solchen Angebot an die Technologie zu gewöhnen. In Zukunft werden immer mehr Kunden über eine eigene Videokonferenz-Ausrüstung (Kamera, Soundsystem und Mikrofon) verfügen und bei einem gleichzeitigen Ausbau der verfügbaren Bandbreiten wird dies dazu führen, daß die audiovisuelle Kommunikation ein unverzichtbarer Bestandteil eines professionellen InternetAuftritts werden wird.

\section{Literaturverzeichnis}

Anderer, B. (1998): Wer bist Du? Sichere Identifikation im World Wide Web. Bank und Markt, Heft 31998

Birkelbach, J. (1998): Rein oder nicht rein? Internet-Banking zwischen Vision und Krise. c't. 9/98. S. 90-91.

Booz Allen \& Hamilton (1997): Internet Banking in Europe: A Survey of Current Use an Future Prospects. Studie 1997.

Buhl, H.U./Visser V.; Will A. (1998): Visualisierung des Bankgeschäfts. in: Weinhardt, C., Meyer zu Selhausen, H., Morlock, M., Hrsg., Informationssysteme in der Finanzwirtschaft, Springer, Berlin, 1998, S. 73-89.

Damaschke, G. (1998): Design. Web-to-date. Global Online. 1-2/98. S. 40-43.

Disterer, G./Reimers-Mortensen, S. (1997): Strategische Optionen für Direktbanken. Die Bank. 3/97. S. 132-139.

Dohmen, M. (1998): Vom Anruf zum Kontakt. Agenten auf Knopfdruck. Net Investor. 6/98. S. 46-47.

Knierim, U. (1997): Online-Callcenter. Rückrufaktion. Global Online. 12/97. S. 60-61.

Lange, Th. A. (1998): Internet Banking. Der Bankvertrieb im Umbruch, Wiesbaden 1998.

Rahlenbeck, E. (1997): Versicherung. Wenn Herr Kaiser online grüßt. Global Online. 8/97. S.28-31.

Radetzky, G. v. (1998): Internet Call-Center. König Kunde auf allen Kanälen. Net Investor. 6/98. S. 44-45.

Röver, S./Schlumpberger A.: The SRT Protocol - Die SET-Erweiterung von Brokat. www.brokat.com/cgi/infopool/link.pl?0+technet+1005

Schmeh, K. (1997): Datenverschlüsselung im Internet: Abhörgefahr gebannt? bank und markt, Heft 81997 
Scholz, C. et al. (1995): Virtuelle Banken - Eine Projektskizze. Diskussionspapier der Untergruppe "Virtuelle Banken" im Rahmen des Schmalenbach-Arbeitskreises "Unternehmerische Partnerschaften". www.orga.uni-sb.de/_archiv/publikationen/banken.html.

Unisys (1996): Internet Banking in Europe. Internet Magazine. Dec. 1996.

The Yankee Group (1998): Cyber Call-Centers 1998: From Internet Enabled to Internet Architected Call Centres, Telecommunications REPORT Vol. 13, No. 3, Feb. 1998 